\title{
The confinement of an annealed branched polymer by a potential well
}

\author{
Alexander Y. Grosberg ${ }^{1}$, Joshua Kelly ${ }^{2}$, and Robijn Bruinsma ${ }^{2,3}$ \\ ${ }^{1}$ Department of Physics and Center for Soft Matter Research, New York University \\ 4 Washington Place, New York NY 10003, USA \\ E-mail: ayg1@nyu.edu \\ ${ }^{2}$ Department of Physics and Astronomy, University of California, Los Angeles, CA 90095, USA \\ ${ }^{3}$ Department of Chemistry and Biochemistry, University of California, Los Angeles, CA 90095, USA
}

Received July 27, 2016, published online November 25, 2016

\begin{abstract}
The Lifshitz equation for the confinement of a linear polymer in a spherical cavity of radius $R$ has the form of the Schrödinger equation for a quantum particle trapped in a potential well with flat bottom and infinite walls at radius $R$. We show that the Lifshitz equation of a confined annealed branched polymer has the form of the Schrödinger equation for a quantum harmonic oscillator. The harmonic oscillator potential results from the repulsion of the many branches from the potential walls. Mathematically, it must be obtained from the solution of the equation of motion of a second, now classical, particle in a non-linear potential that depends self-consistently on the eigenvalue of the quantum oscillator. The resulting confinement energy has a $1 / R^{4}$ dependence on the confinement radius $R$, in agreement with scaling arguments. We discuss the application of this result to the problem of the confinement of single-stranded RNA molecules inside spherical capsids.
\end{abstract}

PACS: 36.20.-r Macromolecules and polymer molecules;

87.15.H- Dynamics of biomolecules.

Keywords: confined branched polymer, Lifshitz equation.

\section{Introduction}

I.M. Lifshitz showed in 1968 that the problem of the confinement of an ideal linear polymer chain by a potential well at finite temperatures can be mapped onto that of the capture of a quantum particle by a potential well at zero temperature $[1,2]$. He formulated the concept of ground state dominance, which states that if the groundstate of the quantum particle is part of a discrete spectrum then the polymer problem maps onto this groundstate in the limit of long polymer chains. Specifically, the Green function $G_{N}(\mathbf{r})$ of the polymer - the partition sum of an $N$-segment chain with endpoints separated by $\mathbf{r}$ - can be expressed as a bilinear expansion over the eigenfunctions of the corresponding Schrödinger operator. The mathematical singularity of the generating function $G(p, \mathbf{r})=\sum_{N} p^{N} G_{N}(\mathbf{r})$ closest to the origin in the complex $p$ plane is an isolated simple pole at $p=p^{*}$ for a confined linear polymer. If $\Psi(\mathbf{r})$ is the solution of the Schrödinger equation for the groundstate wavefunction then the singular part of the generating function has the form $G(p, \mathbf{r}) \propto \Psi(\mathbf{r}) /\left(p-p^{*}\right)$ with $1 / p^{*}$ the boundstate energy eigenvalue. Dominance of this pole when the inverse transform is performed to obtain $G_{N}(\mathbf{r})$ is equivalent to ground state dominance, with $G_{N}(\mathbf{r}) \propto \Psi(\mathbf{r}) \exp \left(-N / p^{*}\right)$.

The Lifshitz method proved fruitful and has led to many developments and applications (for a review, see [3]). However, generalizing his approach to more complex polymeric systems, such as heteropolymers or branched polymers, has proven to be far from trivial. Here, we will focus on the confinement of annealed branched polymers, i.e., branched polymers whose geometric structure is subject to thermal fluctuations [4]. The associated entropic free energy is an important contribution to the total free energy. The large, single-stranded RNA molecules that constitute the genomes of RNA viruses ("vRNA" molecules) can be viewed as realizations of annealed branched polymers [5]. These vRNA molecules can adopt a very large number of branched "secondary structures" that are within $k_{B} T$ of the groundstate, as demonstrated by microscopy studies of vRNA molecules in solution [6]. Other 
studies demonstrated the flexibility of the secondary structure: if vRNA molecules are confined then their secondary structure becomes increasingly branched [7]. The physics of the confinement of vRNA molecules inside a potential well, including the manner their secondary structure adapts to a confining potential, is an important issue for a physical description of the process of viral assembly, with the confining potential representing the interaction of the RNA molecule with the capsid proteins [8]. In the following we will consider the problem of the confinement of an annealed branched polymer inside a spherical potential well of radius $R$ as a (very) simple model of the physics of encapsidation of vRNA molecules by viral capsid proteins.

\section{Lifshitz theory of confined annealed branched polymers}

\subsection{Basic equations}

The formulation of Lifshitz theory for the case of annealed branched polymer was developed in Ref. 9. There, it was shown that the mathematical singularity of the generating function closest to the origin is a branch point instead of a simple pole. For the simple case of a branched polymer composed of monomers that can only be endpoints or triple branching points (see Fig. 1), the generating function adopts the form $G(p, \mathbf{r}) \simeq \Phi(\mathbf{r})+\Psi(\mathbf{r})\left(p-p^{*}\right)^{1 / 2}$. Instead of the single Schrödinger equation that suffices for linear chains, Lifshitz theory now requires the solution of two coupled equations for the two unknown functions $\Phi(\mathbf{r})$ and $\Psi(\mathbf{r})$. The physical interpretation of the two fields is as follows. The spatial distribution of end-points is proportional to $\mathrm{e}^{-\varphi_{1}} \Psi$, where $\varphi_{1}$ is an external potential acting only on the endpoints in units of the thermal energy $k_{B} T$. The spatial distribution of the branch-points is proportional to $e^{-\varphi_{3}} \Phi^{2} \Psi$, where $\varphi_{3}$ is an external potential acting only

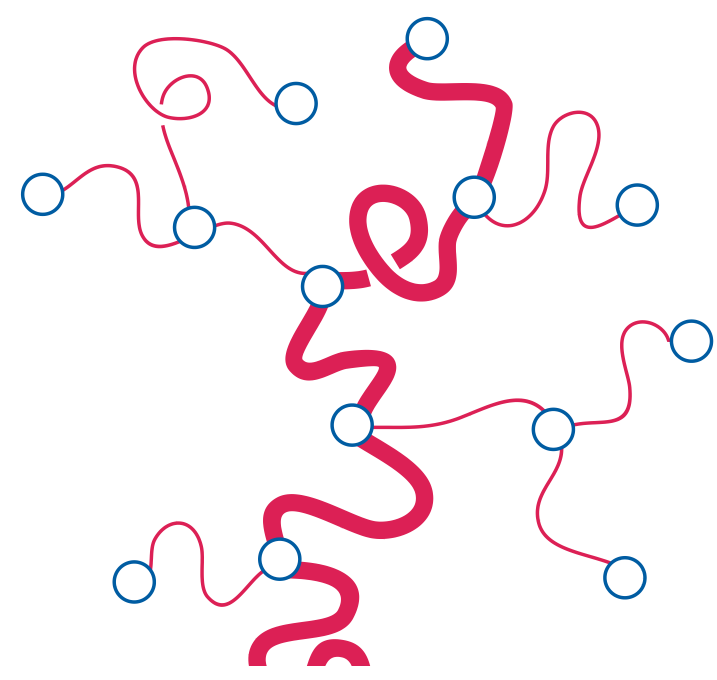

Fig. 1. (Color online) Branched polymer composed of $N=14$ monomers that are either endpoints of branch points. The heavy line shows an arbitrary choice for the "backbone" chain. on the branch points [9]. As an example, the branching function $\Phi$ for a Cayley tree or dendrimer should be large in the center of the tree and small at its periphery while $\Phi$ should be a constant for a polymer composed of a linear backbone with single-monomer side branches.

The equations for the two fields have the form

$$
\begin{gathered}
\left(1 / p^{*}\right) \Phi(\mathbf{r})=\Lambda_{1} \hat{g} \mathrm{e}^{-\varphi_{1}(\mathbf{r})}+\Lambda_{3} \hat{g}\left[\mathrm{e}^{-\varphi_{3}(\mathbf{r})} \Phi^{2}(\mathbf{r})\right], \\
\left(1 / p^{*}\right) \Psi(\mathbf{r})=2 \Lambda_{3} \hat{g}\left[\mathrm{e}^{-\varphi_{3}(\mathbf{r})} \Phi(\mathbf{r}) \Psi(\mathbf{r})\right]
\end{gathered}
$$

where $\Lambda_{1}$ and $\Lambda_{3}$ are the fugacities of the end-points and branch-points, respectively. The zero of the potentials is chosen such that $\left.\varphi_{1,3}(\mathbf{r})\right|_{|\mathbf{r}| \rightarrow \infty}=0$. Next, $\hat{g}$ is an integral operator that describes flexible bonds between monomers. Following Ref. 1, we use $\hat{g}=\exp \left[\frac{a^{2}}{2 D} \nabla^{2}\right]$ with $a$ the length scale of a monomer-monomer bond, $D$ the dimension of space, and $\nabla^{2}$ the Laplace operator. As before, $1 / p^{*}$ plays the role of the groundstate energy eigenvalue. After $p^{*}$ has been determined, the non-translational part of the free energy of the macromolecule is obtained from $\Omega=N \ln p^{*}$ with $N$ the total number of monomers.

For example, for unconfined branched polymers that are freely fluctuating in space, the external potentials can be set to zero and the two fields $\Phi$ and $\Psi$ can be assumed to be uniform [9]. Solving Eqs. (1) is then straightforward, leading to $p_{0}^{*}=1 /\left[2\left(\Lambda_{1} \Lambda_{3}\right)^{1 / 2}\right]$, so the free energy of a free annealed branched polymer equals $\Omega_{\text {free }}=-N \ln \left[2\left(\Lambda_{1} \Lambda_{3}\right)^{1 / 2}\right]$, which reproduces earlier results for annealed branched polymers in the absence of applied fields [4].

Returning to the general case, if one approximates $\hat{g} \simeq 1+\frac{a^{2}}{2 D} \nabla^{2}$ then Eq. (1b) has the form of a linear Schrödinger-like equation similar to the Lifshitz equation for linear polymers [1]. The difference is that the monomers now are subject to an effective potential that depends on the branching function $\Phi$. One could interpret Eq. (1b) as the Lifshitz equation for one (arbitrary chosen) linear backbone of the branched polymer (see Fig. 1) that "feels" an effective potential communicated to the backbone via the tentacle-like branches that extend into the surrounding environment. On the other hand, Eq. (1a) for the branching function $\Phi$ is non-linear and specific for branched polymers.

It is instructive at this point to compare the qualitative features of Eqs. (1) with the predictions of scaling theory for the confinement of polymers in a spherical potential well of radius $R$. According to scaling theory, the confinement free energy cost of a polymer should have the general form $k_{B} T f\left(R / R_{0}(N)\right)$ with $f(x)$ a dimensionless scaling function and $R_{0}(N)$ the radius of gyration for 


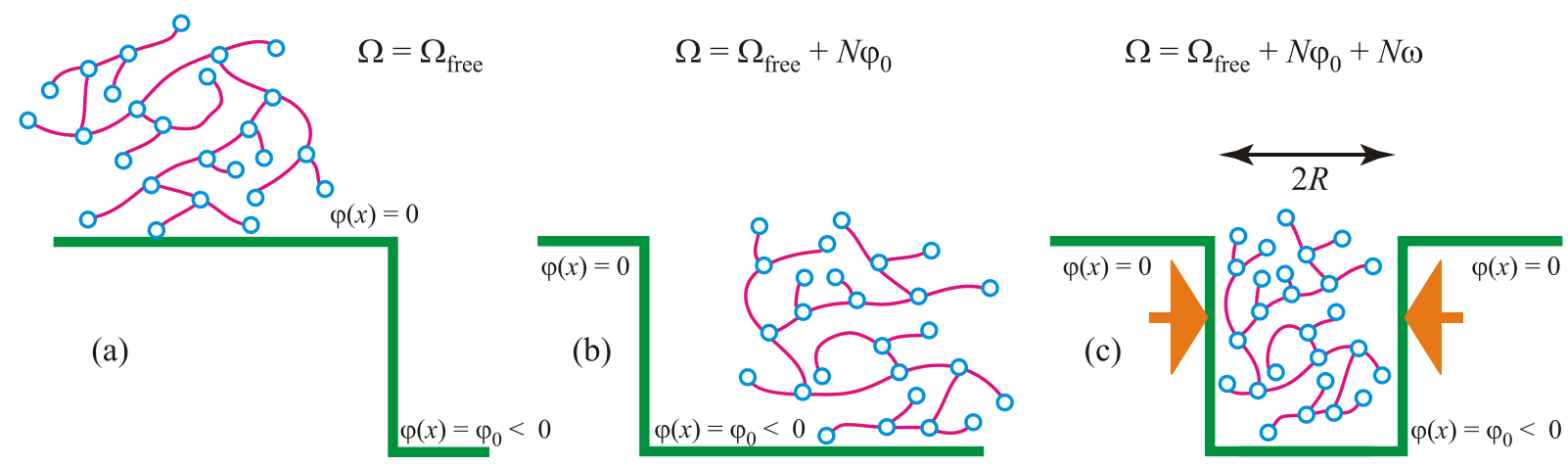

Fig. 2. (Color online) Confinement of a branched polymer. In the leftmost figure (a), the branched polymer is far from the potential well. It has a free energy $\Omega_{\text {free }}$. In the middle figure (b), the entire polymer is placed at the bottom of a deep and very wide potential with a potential energy per monomer $\varphi_{0}<0$. The total free energy is now $\Omega_{\text {free }}+N \varphi_{0}$. Finally, in the rightmost figure (c), the size of the well is reduced laterally so the polymer is compressed. The compression work is equal to $\omega$ per monomer. The total free energy is now $\Omega_{\text {free }}+N \varphi_{0}+N \omega$ (see Eq. (4)).

the free (unconfined) polymer. The scaling function is determined by the condition that if the confinement radius $R$ is small compared to $R_{0}(N)$ then the confinement free energy should be extensive and thus proportional to the number of monomers $N$. The radius of gyration $R_{0}(N)$ of an unconfined ideal annealed branched polymer was long ago shown to have the form $R_{0}(N) \simeq a N^{1 / 4}$ [10]. It follows that for annealed branched polymers the scaling function must depend on $x$ as $f(x) \propto 1 / x^{4}$ for small $x$. This, in turn, means that the confinement free energy should scale as $N(a / R)^{4}$.

It is however unclear how such an answer could emerge from Eqs. (1) since the Schrödinger equation for a particle confined in a box of radius $R$ has a groundstate energy proportional to $1 / R^{2}$, not $1 / R^{4}$. One of the goals of the present paper is to resolve this apparent contradiction.

\subsection{Reducing the equations}

To simplify the problem, we first eliminate $\Lambda_{1}$ and $\Lambda_{3}$. We need to find the contribution $\Omega-\Omega_{\text {free }}=N \ln \left(p^{*} / p_{0}^{*}\right)$ to the thermodynamic potential that results from turning on the external fields. This motivates us to introduce the quantities $q^{*}=2 p^{*} \sqrt{\Lambda_{1} \Lambda_{3}}$ and $\tilde{\Phi}=\sqrt{\Lambda_{3} / \Lambda_{1}} \Phi$. In terms of these reduced quantities, Eqs. (1) adopt a form that no longer depends on $\Lambda_{1}$ and $\Lambda_{3}$ :

$$
\begin{gathered}
\left(2 / q^{*}\right) \tilde{\Phi}(\mathbf{r})=\hat{g}\left[\mathrm{e}^{-\varphi_{1}}+\mathrm{e}^{-\varphi_{3}} \tilde{\Phi}^{2}(\mathbf{r})\right], \\
\left(1 / q^{*}\right) \Psi\left((\mathbf{r})=\hat{g}\left[\mathrm{e}^{-\varphi_{3}} \tilde{\Phi}(\mathbf{r}) \Psi(\mathbf{r})\right] .\right.
\end{gathered}
$$

From here on we will work with these reduced equations, dropping the tilde sign for simplicity. Next, assume a spherical potential well that acts in the same way on both monomer types so

$$
\varphi_{1}(\mathbf{r})=\varphi_{3}(\mathbf{r})=\varphi(\mathbf{r})=\left\{\begin{array}{lll}
0 & \text { if } & |\mathbf{r}|>R \\
\varphi_{0} & \text { if } & |\mathbf{r}|<R
\end{array}\right.
$$

where $\varphi_{0}<0$ is the depth of the potential well. The (nontranslational) free energy of a polymer bound in a deep well (i.e., with $\left|\varphi_{0}\right| \gg>1$ ) and a radius large compared to the unperturbed radius of gyration is then $\Omega_{\text {free }}+N \varphi_{0}$. We will assume that the radius $R$ is less than the unconfined radius of gyration $R_{0}(N)$. Under conditions of ground state dominance, this should increase the free energy by an amount proportional to $N$ :

$$
\Omega=\Omega_{\text {free }}+N \varphi_{0}+N \omega .
$$

The dimensionless quantity $\omega$, which is positive by construction and small compared to one for $R / a>1$ (i.e., $0<\omega<<1$ ), is the confinement free energy per monomer that we need to determine (see also Fig. 2). It is related to $q^{*}$, the inverse eigenvalue, by

$$
q^{*}=\mathrm{e}^{\varphi_{0}} \mathrm{e}^{\omega} \simeq \mathrm{e}^{\varphi_{0}}(1+\omega)
$$

We can eliminate $q^{*}$ and re-write Eqs. (2) in the form

$$
\begin{gathered}
2(1-\omega) \Phi=\hat{g}\left[\mathrm{e}^{\varphi_{0}-\varphi}\left(1+\Phi^{2}\right)\right], \\
(1-\omega) \Psi=\hat{g}\left[\mathrm{e}^{\varphi_{0}-\varphi} \Phi \Psi\right]
\end{gathered}
$$

that we will use in the following.

It would appear reasonable to assume that $\Phi$ is approximately equal to a constant outside the well and another constant inside the well. Denote the two constants by $\Phi_{\text {out }}$ and $\Phi_{\text {in }}$, respectively. Under this assumption

$$
\begin{gathered}
2 \mathrm{e}^{-\varphi_{0}}(1-\omega) \Phi_{\text {out }} \simeq 1+\Phi_{\text {out }}^{2}, \\
2(1-\omega) \Phi_{\text {in }} \simeq 1+\Phi_{\text {in }}^{2},
\end{gathered}
$$

where we set $\hat{g} \rightarrow 1$. For $\mathrm{e}^{-\varphi_{0}}>>1$, the quadratic equation (7a) for the outside has a real solution with $\Phi_{\text {out }} \sim \mathrm{e}^{\varphi_{0}} \rightarrow 0$ for a deep well. On the other hand Eq. (7b) has no real solutions for $\omega>0$. Because of the physical meaning of $\Phi$, 
complex solutions are unphysical. This means that the assumption that $\Phi$ is almost constant inside the well has to be abandoned. To find the actual spatial profile of $\Phi$, we need to solve the non-linear differential equation

$$
-[1-\Phi]^{2}-2 \omega \Phi \simeq \frac{a^{2}}{2 D} \nabla^{2} \Phi^{2}
$$

where we assumed $\hat{g} \simeq 1+\frac{a^{2}}{2 D} \nabla^{2}$. Note that because $\Phi$ is a positive real number, the left hand side is strictly negative. This confirms that Eq. (7b) has no solution.

\subsection{Solution for $\Phi(x)$ in $D=1$}

We first consider the one-dimensional $(D=1)$ case, which describes a branched polymer confined between two parallel plates a distance $2 R$ apart. The origin $x=0$ is at the midpoint between the plates. For $D=1$, Eq. (8) for $\Phi(x)$ has the mathematical form of the equation of motion of a classical particle with $x / a$ as the effective (dimensionless) time and $\Phi^{2}(x)=y$ as the effective position. The equation of motion

$$
\begin{aligned}
\ddot{y} & =-2[1-\sqrt{y}]^{2}-4 \omega \sqrt{y}= \\
& =-\frac{\partial}{\partial y}[\underbrace{y^{2}+2 y-\frac{8}{3} y^{3 / 2}+\frac{8 \omega}{3} y^{3 / 2}}_{U(y)}] .
\end{aligned}
$$

is that of a particle moving without friction in the "potential energy" landscape $U(y)$ shown in Fig. 3. For positive $\omega$, the potential energy $U(y)$ is a monotonically increasing function of $y$, although the slope does become very small for $y \simeq 1$ for $\omega$ small compared to one. This equation of

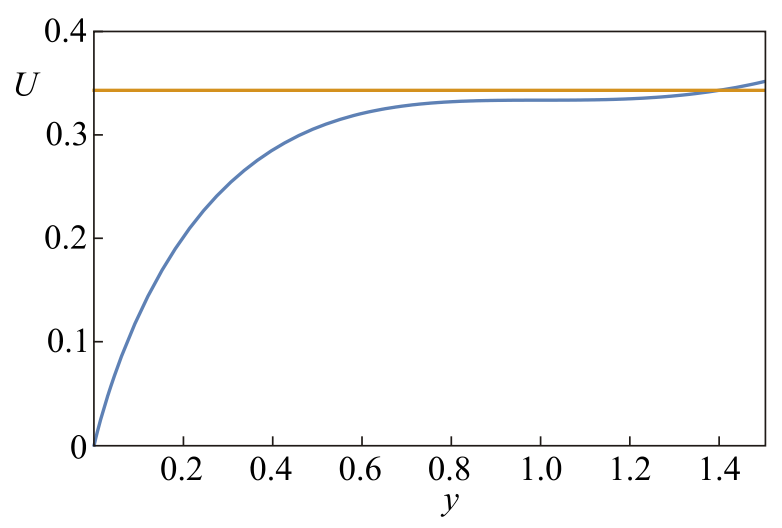

Fig. 3. (Color online) Plot of the effective potential energy $U(y)$ for the equivalent particle equation of motion of Eq. (9) for $\omega=10^{-4}$. The horizontal line is effective mechanical energy $U\left(y_{0}\right)$, which is the first integration constant of the equation of motion. For the sake of visibility, this line is shown for $\delta=y_{0}-1=0.4$, which is significantly larger than the actual solution. motion must be solved under the boundary conditions that the particle has zero "velocity" $\dot{y}=0$ at $x=0$ (because of the $x \leftrightarrow-x$ symmetry), while at $x=R$ the "position" $y(R / a)$ is zero since $\Phi_{\text {out }} \rightarrow 0$ at the two plates (see Eq. (7a)).

Integrating Eq. (9) subject to the second boundary condition gives

$$
\frac{x}{a}=-\int_{y_{0}}^{y} \frac{d y^{\prime}}{\sqrt{2\left(U\left(y_{0}\right)-U\left(y^{\prime}\right)\right)}},
$$

where $y_{0}=\Phi(0)^{2}$ is the value of $y(x)$ at $x=0$. Here, $y_{0}$ plays the role of an integration constant that must be determined by the first boundary condition $y(R / a)=0$ at $x=R$ :

$$
\frac{R}{a}=\int_{0}^{y_{0}} \frac{d y^{\prime}}{\sqrt{2\left(U\left(y_{0}\right)-U\left(y^{\prime}\right)\right)}} .
$$

For $y_{0}=1$ and $\omega=0$, the integral in Eq. (11) diverges at its upper limit, since both first and second derivative of $U(y)$ vanish at $y=1$ when $\omega=0$. In particle language, the particle takes an infinite amount of time to reach $y=1$ with its velocity decreasing to zero over time. If $R / a$ is large but finite then $\omega$ is a small positive number while $y_{0}$ is slightly larger than one. The particle still spends most of its time near $y=1$ where its effective velocity is low but the integral no longer diverges. Write $y_{0}=1+\delta$ with $0<\delta<<1$. There are now two important small dimensionless parameters: $\delta$ and the confinement free energy per monomer $\omega$. One does not know in advance the relative magnitudes of these two small parameters. Assume for now that $\omega \sim \delta^{2}$, which is to be verified in the following.

For $R / a>>1$, the integral in Eq. (11) is dominated for $y$ values in the vicinity of the upper limit $y \simeq y_{0} \simeq 1$. It can be evaluated approximately by expanding $U(y)$ in a Taylor series around $y=y_{0}$. It is necessary to go to third order in $y_{0}-y$ because both first and second derivatives are small in this regime:

$$
\begin{aligned}
U\left(y_{0}\right)-U(y) \simeq & \frac{\delta^{2}+8 \omega}{2}\left(y_{0}-y\right)-\frac{\delta}{2}\left(y_{0}-y\right)^{2}+ \\
& +\frac{1}{6}\left(y_{0}-y\right)^{3}+\ldots,
\end{aligned}
$$

assuming $\delta<<1$ and $\omega \sim \delta^{2}$. Next, change the integration variable in Eq. (11) to $\eta=\left(y_{0}-y^{\prime}\right) / \sqrt{3\left(\delta^{2}+8 \omega\right)}$ and introduce the new quantity

$$
\gamma=\frac{\sqrt{3} \delta}{\sqrt{8 \omega+\delta^{2}}} .
$$

For $\omega \sim \delta^{2}, \gamma$ should be of the order of one. In the next section, we will show that $\gamma$ is indeed a numerical constant of the order of unity in that limit. For now, assume that that is the case. 
Under that assumption, Eq. (11) reduces to

$$
\frac{R}{a} \simeq \frac{\sqrt{\gamma} \rho(\gamma)}{\sqrt{\delta}}-2 \sqrt{3}+\ldots
$$

Here

$$
\rho(\gamma)=\int_{0}^{\infty} \frac{d \eta}{\sqrt{\eta-\gamma \eta^{2}+\eta^{3}}}
$$

is a smoothly-varying function of its argument $\gamma$. As discussed in Appendix A, $\rho(\gamma)$ is of the order of one for $\gamma$ of the order of one. The first term of Eq. (14) diverges in the limit of $\delta \rightarrow 0$. The second term is of course a constant while the dots stand for terms that vanish in that limit. It follows that if $\gamma$ is a numerical constant of the order of one, then $\delta \sim(a / R)^{2}$ to leading order in the limit $\delta \rightarrow 0$. Next, eliminate $\delta$ from Eq. (14) in favor of $\gamma$ and $\omega$ using Eq. (13). After rearrangement, one obtains an expression for the confinement free energy per monomer $\omega$ in terms of the plate separation $2 R$ :

$$
\omega(R) \simeq \frac{A(\gamma)}{(R / a+2 \sqrt{3})^{4}},
$$

where $A(\gamma)=\left(3-\gamma^{2}\right) \rho(\gamma)^{4} / 8$. Note that this expression is consistent with the scaling prediction $\omega \sim 1 / R^{4}$ for $R / a \rightarrow \infty$.

The $\Phi(x)$ profile can now be determined as well. Performing the same manipulations on Eq. (10) as was done for Eq. (11) and assuming the same limits gives

$$
\frac{x}{a} \simeq \frac{\sqrt{\gamma}}{\sqrt{\delta}} \int_{0}^{\eta} \frac{d \eta^{\prime}}{\sqrt{\eta^{\prime}-\gamma \eta^{\prime 2}+\eta^{\prime 3}}}
$$

In the central part of the well, where $\eta \simeq\left(y-y_{0}\right) / \delta$ is small compared to one, one can neglect the second and third term under the square root in the integrand, yielding $\eta(x) \simeq \frac{\delta}{4 \gamma}\left(\frac{x}{a}\right)^{2}$. The corresponding profile for $\Phi(x)$, given by

$$
\left.\Phi(x)\right|_{x<<R} \simeq 1+\frac{\delta}{2}-\frac{3}{8}\left(\frac{\delta}{\gamma}\right)^{2}\left(\frac{x}{a}\right)^{2}
$$

is then parabolic. Equation (18) must break down close to the borders $|x|=R$ of the potential well, as can be seen from the fact that $\Phi(|R|)$ is close to one according to Eq. (24) while in actuality $\Phi(\mid R) \mid \sim \mathrm{e}^{\varphi_{0}} \rightarrow 0$. In order to find $\Phi(x)$ closer to the borders, it is convenient to express it in the form of a Taylor expansion as $\Phi(x)=1+\sum_{k=0}^{\infty} \beta_{k}(x / a)^{2 k}$. Only terms even in $x$ enter because of the mirror symmetry $x \leftrightarrow-x$ of the well. Inserting the expansion into Eq. (8) for $\Phi(x)$ yields a recurrence relations for the coefficients $\beta_{k}[11]$ :

$$
\begin{gathered}
\beta_{1}=-\frac{\frac{1}{2} \beta_{0}^{2}+\omega+\omega \beta_{0}}{1+\beta_{0}}, \\
\left.\beta_{k+1}\right|_{k \geq 1}=-\frac{\frac{2 \omega \beta_{k}+\sum_{p=0}^{k} \beta_{p} \beta_{k-p}}{(k+1)(2 k+1)}+\sum_{p=1}^{k} \beta_{p} \beta_{k-p+1}}{2\left(1+\beta_{0}\right)} .
\end{gathered}
$$

Starting from $\beta_{0}=(1+\delta)^{1 / 2}-1 \simeq \delta / 2$, the other coefficients can be obtained to any order. Including terms up to $k=3$ gives:

$$
\begin{aligned}
\Phi(x)= & 1+\delta / 2-\frac{3}{8} \frac{\delta^{2}}{\gamma^{2}}\left(\frac{x}{a}\right)^{2}+\frac{1}{32} \frac{\delta^{3}}{\gamma^{2}}\left(\frac{x}{a}\right)^{4}- \\
& -\frac{3}{640} \frac{\delta^{4}}{\gamma^{4}}\left(1+\frac{2}{9} \gamma^{2}\right)\left(\frac{x}{a}\right)^{6}+\ldots
\end{aligned}
$$

where we used Eq. (13) for $\gamma$ to eliminate $\omega$. In each term we included only the lowest power of $\delta$ that was non-zero. It is easy to show that in the large $k$ limit, $\beta_{k} \sim k \delta^{k+1}$ asymptotically so the series converges rapidly provided $\delta(x / a)^{2}<<1$. We now turn to the proof that $\gamma$ indeed is a purely numerical factor.

\subsection{Solution for $\Psi(x)$ in $D=1$}

The constancy of $\gamma$ will be demonstrated by solving Eq. (6b) for $\Psi$. In $D=1$ this equation is given by

$$
(1-\omega) \Psi(x) \simeq\left(1+\frac{a^{2}}{2} \frac{d^{2}}{d x^{2}}\right)[\Phi(x) \Psi(x)],
$$

for $|x|<2 R$. If we introduce $\chi(x)=\Psi(x) \Phi(x)$ then this equation adopts the form of the Schrödinger equation

$$
-\frac{a^{2}}{2} \frac{d^{2} \chi}{d x^{2}}+\frac{1-\omega}{\Phi(x)} \chi \simeq \chi,
$$

with an effective potential energy determined by the branching function $\Phi$.

\subsubsection{Harmonic approximation}

If we use the parabolic form Eq. (18) in Eq. (22) then one obtains the Schrödinger equation for a $D=1$ harmonic oscillator:

$$
\left[-\frac{a^{2}}{2} \frac{d^{2}}{d x^{2}}+\frac{3}{8}\left(\frac{\delta}{\gamma}\right)^{2}\left(\frac{x}{a}\right)^{2}\right] \chi=\frac{\delta}{2} \chi,
$$


with the unusual feature that the spring constant depends on the eigenvalue $\delta / 2$. Using the well-known energy eigenvalue of the groundstate of the harmonic oscillator it follows, after some straightforward re-naming of variables, that $\gamma=\sqrt{3} / 2$. From Eq. (13) it then follows that our initial assumption $\omega \simeq \delta^{2}$ is confirmed. For $\gamma=\sqrt{3} / 2$ the amplitude $A(\gamma)$ in the scaling relation Eq. (16) equals $A \approx 87.6$.

The function $\chi(x)$ is proportional to $\exp \left[-\frac{\sqrt{3} \delta}{4 \gamma} \frac{x^{2}}{a^{2}}\right]$ from which it follows that the endpoint profile $\Psi(x)$ has a Gaussian dependence on $x /(R+2 \sqrt{3} a)$ :

$$
\Psi(x) \propto \exp \left[-\frac{1}{2 \sigma^{2}}\left(\frac{x}{R+2 \sqrt{3} a}\right)^{2}\right]
$$

with a standard deviation $\sigma=1 / \sqrt{\gamma} \rho(\gamma)$ that is a purely numerical factor. If $\gamma=\sqrt{3} / 2$, then $\sigma=0.256$.

\subsubsection{Anharmonic corrections}

In the harmonic approximation, the quantity $\gamma$ is thus indeed a purely numerical factor. The endpoint profile $\Psi(x)$ Eq. (24) obtained in the harmonic approximation decays as a Gaussian on a scale of $R$. At the borders, where the harmonic approximation for $\Psi(x)$ fails, $\Psi(|R|) / \Psi(0)$ is a numerical factor independent of $R$. Since $\Psi(|R|) / \Psi(0)$ does not go to zero in limit of large $R / a$, we need to demonstrate that anharmonicity does not contribute any $R$-dependent terms to $\gamma$.

Insert Eq. (20) into Eq. (22). It is convenient to introduce the dimensionless position variable $z=3^{1 / 4}(\delta / \gamma)^{1 / 2}(x / a)$. The equation then adopts the form of a Schrödinger equation for a $D=1$ anharmonic oscillator

$\left[-\frac{d^{2}}{d z^{2}}+\frac{1}{4} z^{2}-\frac{\gamma}{48 \sqrt{3}} z^{4}+\frac{2 \gamma^{2}+9}{8640} z^{6}+\ldots.\right] \chi=\frac{\gamma}{\sqrt{3}} \chi$

After the rescaling, this equation no longer depends on $\delta$. Since $\beta_{k} \sim k \delta^{k+1}$, this cancelation of $\delta$ takes place to all orders in $k$. Thus, $\gamma$ is the only unknown in Eq. (25), which shows that $\gamma$ remains a purely numerical factor when anharmonicity is included. As before, the potential energy of the anharmonic oscillator depends on the groundstate eigenvalue $(\gamma / \sqrt{3})$. When the groundstate eigenvalue is evaluated, this results in a self-consistency condition for the value of $\gamma$ so $(\gamma / \sqrt{3})$. The result no longer equals $1 / 2$ but this change in the numerical value for $\gamma$ does not change the scaling exponent in Eq. (16) for $\omega$, though it will of course change the value of the prefactor $A(\gamma)$. The actual numerical value of $\gamma$, determined numerically remains close to $\sqrt{3} / 2$.
In summary, the confinement free energy of an annealed branched polymer between two plates with a separation of $2 R$ decreases with $R$ in agreement with the scaling prediction that it should be proportional to $1 / R^{4}$ in the large $R$ limit. The branches of the polymer generate an approximately harmonic self-consistent potential for the backbone chain, which result in a Lifshitz equation that has the mathematical form of the Schrödinger equation for a $D=1$ quantum harmonic oscillator.

\section{Numerical solutions}

In this section we discuss numerical solutions of Eqs. (1). We first verify our analytical result Eq. (16) for the $D=1$ potential well and then consider the case of a $D=3$ potential well, which does not allow analytical integration. In this section, the integral operator $\hat{g}=\exp \left[\frac{a^{2}}{2 D} \nabla^{2}\right]$ is kept in its complete form without expansion. Technical details of our numerical methods are presented in Appendix B.

\subsection{Numerical solution: $D=1$}

Figure 4 shows the numerically computed confinement free energy per monomer $\omega$ as a function of the well size $R / a$. The well depth $\varphi_{0}$ was equal to -10 in units of $k_{B} T$. The dots in Fig. 4 show the numerically computed values, while the dashed (red) line is the result of a fit of the numerical results to the expression $\omega=\frac{A}{(R / a+2 \sqrt{3})^{4}}$, with $A$ treated as a fitting parameter. The fitted value $A \simeq 94.2$ [12] should be compared with the analytical result $A \simeq 87.6$ (see Eq. (16)). The discrepancy is due, presumably, to the fact that the analytical result was obtained in the limit of small $\delta$ while the numerical solution was obtained for $\delta \simeq 0.04$ (or larger).

Next, we show in Fig. 5 the numerically computed endpoint density profile $\Psi(x)$ and branching function $\Phi(x)$ for a $D=1$ potential well with $R / a=15$ and $\kappa_{0}=-10$ and

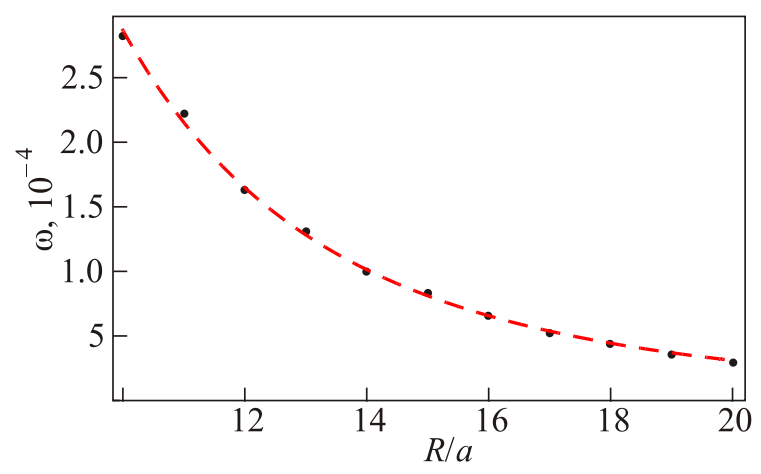

Fig. 4. (Color online) Confinement free energy per monomer $\omega$ as a function of well size $R / a$ in $D=1$. Dots: numerically computed values. Red dashed line: best fit of the form $\omega=\frac{A}{(R / a+2 \sqrt{3})^{4}}$ with $A=94.2$. 


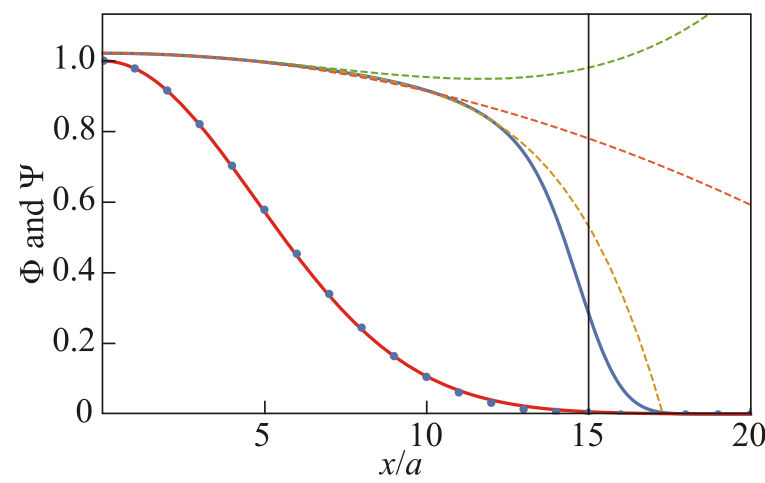

Fig. 5. (Color online) Endpoint density profile $\Psi(x)$ and branching function $\Phi(x)$ for a $D=1$ potential well with $R / a=15$ and $\varphi_{0}=-10$. The numerically computed confinement energy is $\omega \approx 0.00082885$ while $\delta \approx 0.0443$. Dots: numerically computed values for $\Psi(x)$. Lower solid line (red): $\Psi(x)$ according to Eq. (24). The normalization of $\Psi(x)$ is arbitrarily set to $\Psi(0)=1$. Upper solid line (blue): numerically computed $\Phi(x)$. The dashed lines represent quadratic, quartic, and sextic power approximations to $\Phi(x)$ according to Eq. (20). There are no fitting parameters.

compare them with Eq. (24) for $\Psi(x)$ and the analytical Taylor expansion to order $x^{6}$ for $\Phi(x)$ (see Eq. (20)). The sixth-order expansion appears to provide a good approximation for $\Phi(x)$ up to the border of the well at $|x| / a=15$.

For $D=1$, the numerical results are in general consistent with the analytical theory.

\subsection{Numerical solution: $D=3$}

We now turn to the case of the confinement of branched polymers in a radially symmetric well in $D=3$, but keeping with the same parameters $R / a=15$ and $\varphi_{0}=-10$. Figure 6 shows the numerically computed confinement

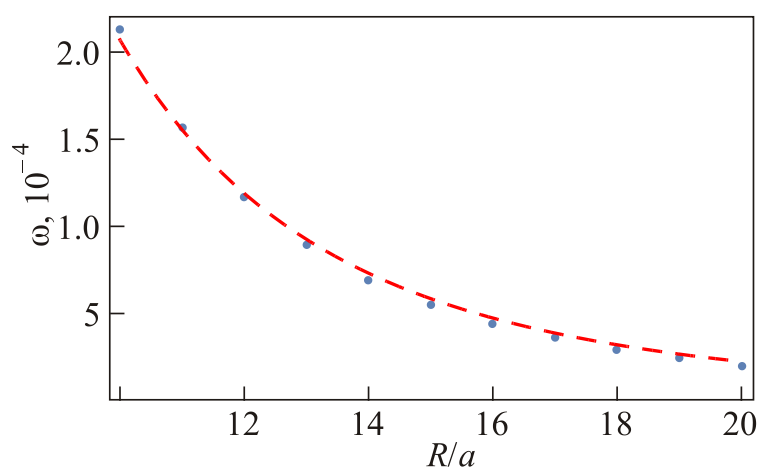

Fig. 6. Confinement free energy per monomer $\omega$ as a function of well size $R / a$ in $D=3$. Dots: numerically computed values. Red dashed line: best fit of the form $\frac{A}{(R / a+C)^{4}}$ with $A=50.6$ and $C=2.41$.

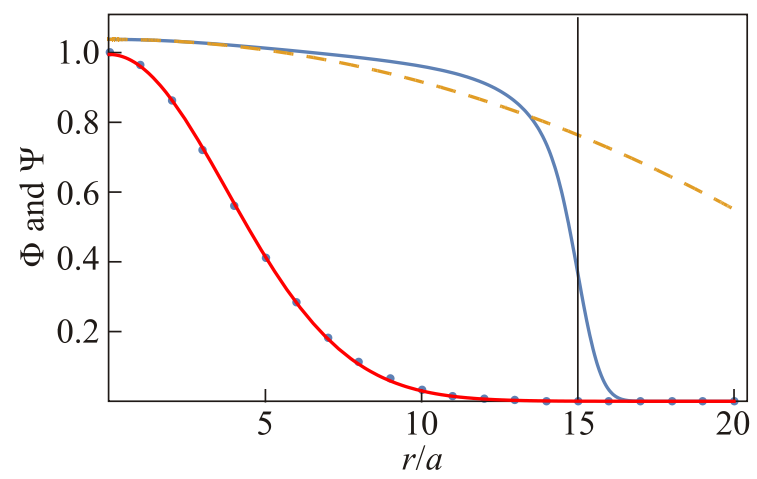

Fig. 7. (Color online) Endpoint density profile $\Psi(r)$ and branching function $\Phi(r)$ for a $D=3$ potential well with $R / a=15$ and $\varphi_{0}=-10$. The numerically computed confinement energy is $\omega \approx 0.000549332$ while $\delta \approx 0.077$. Dots: numerically computed values for $\Psi(r)$. Lower solid line (red): Gaussian fit to these points, with fitted standard deviation $\sigma=3.77$. The normalization of $\Psi(r)$ is arbitrarily set so $\Psi(0)=1$. Upper solid line (blue): numerically computed $\Phi(r)$. The dashed line represents a quadratic approximations to $\Phi(r)=1+\beta_{0}+\beta_{1} r^{2}$, with $\beta_{0}=0.038$ and $\beta_{1}=-0.0013$ according to Eq. (27).

free energy per monomer $\omega$ as it depends on the radius $R$ of the potential well in $D=3$. The dots show again the numerically computed values while the solid line is a fit of the numerical results to the expression $\omega=\frac{A}{(R / a+C)^{4}}$, treating both $A$ and $C$ as fitting parameters.

$$
\text { 3.3. } D=1 \text { versus } D=3
$$

The numerical results for the $D=1$ and $D=3$ cases look very similar. This can be understood as follows. If one again sets $\Phi^{2}(r)=y(r)$ and $\Phi^{2}(0)=y_{0}=1+\delta$ then Eq. (8) for $D=3$ reduces to

$$
-\frac{1}{4}\left(8 \omega+\delta^{2}\right)=\frac{a^{2}}{6} \nabla^{2} y .
$$

The lowest-order solution

$$
\Phi(r) \simeq 1+\frac{\delta}{2}-\frac{r^{2}}{8 a^{2}}\left(8 \omega+\delta^{2}\right)
$$

has the same form as Eq. (18) when $r$ is replaced by $x$. Figure 7 shows that if one uses in this expression the numerically computed values for $\omega$ and $\delta$ then it again provides a reasonable approximation for $\Phi$ in the center of the well. This approximation must be matched to a solution of Eq. (8) that is valid at the boundary of the well where $\Phi(r)$ rapidly drops to zero. Assuming the width of the boundary region to be small compared to $R$, one actually can use the $D=1$ solution for the border region! This gives 


$$
\frac{r-R}{a}=-\int_{0}^{y} \frac{d y^{\prime}}{\sqrt{2\left(U\left(y_{0}\right)-U\left(y^{\prime}\right)\right)}} .
$$

At $r \sim R$, these two limiting expressions have to match. In the matching region, $\Phi(r) \simeq 1-O(\delta)$, which means that the upper limit of integration in (28) can be extended to about $y_{0}$, which yields for $R$ an equation that is similar to the $D=1$ case. Finally, when the parabolic profile Eq. (27) is inserted in the $D=3$ Lifshitz equation, one obtains a Gaussian profile for $\Psi(r)$ and an eigenvalue of the form $\frac{A}{(R / a+C)^{4}}$ though the values for $A$ and $C$ will be different from the $D=1$ case.

\section{Concluding remarks}

In summary, we have shown that the Lifshitz theory of annealed branched polymers reproduces the $1 / R^{4}$ confinement free energy expected from scaling theory instead of the $1 / R^{2}$ confinement free energy of linear polymers. Mathematically, this was due to a renormalization of the externally potential well by the side branches, which generated a harmonic potential at the center of the well. The strength of the potential depends self-consistently on the confinement energy. The effective Lifshitz equation for the backbone of the branched polymer has the form of the Schrödinger equation of a quantum harmonic oscillator, at least for the low-lying levels of the equivalent quantum problem. Higher levels are, presumably, dominated by the "bare" potential and are expected to depend on $R$ as $1 / R^{2}$. The applicability of the $1 / R^{4}$ result relies on groundstate dominance with $N>>(R / a)^{4}$. For $(R / a)^{2}<<N<(R / a)^{4}$, the higher levels of the potnetial will start to dominate and the confinement free energy is expected to depend on $R$ as $1 / R^{2}$ for smaller $N$ (though we have not shown this).

Apart from confirming scaling theory, Lifshitz theory allows us to determine the spatial profiles of the branching function $\Phi(r)$ and the end-point density $\Psi(r)$. The branching function is nearly constant at the center of the well and drops to zero near the boundary while the endpoint density is a Gaussian with a width of the order of the size of the potential well. Recall that the corresponding $\Psi(x)$ profile for a linear polymer confined between two plates separated by $2 R$ is proportional to $\cos (\pi x / 2 R)$ [1]. The monomers of a linear polymer are thus less confined to the center of the well than the monomers of branched polymer, which is consistent with an increased confinement energy in the large $R$ limit for linear polymers: branching apparently causes an increase in density at the center of the well.

The fact that the branching function is nearly constant over most of the interior of a spherical well while dropping to zero at the edges suggests that branched polymers adopt a dendrimer-like configurations. This is reasonable since a dendrimer configuration provides maximum compaction.
It also agrees with the experiments on the confinement of viral RNA molecules. In solution, these molecules form rather elongated "branched backbone" structures while under confinement they resemble dendrimers with the endpoints extending outwards [7]. One would expect that for larger well radii $R$, one should encounter more branched backbone configurations with a more uniform branching function profile, since that might provide a higher configurational entropy. Though we did not encounter such a transition, it may be associated with the breakdown of groundstate dominance for increasing $R$ when $N \simeq(R / a)^{4}$. It would be interesting to include the higher excited states of the quantum oscillator to see if this transition could be brought out using the Lifshitz formalism.

We conclude by noting that the neglect of excluded volume interactions is a limitation that is even more serious for branched polymers than for linear polymers because branching increases the monomer density. Interestingly, the confinement free energy of annealed branched polymers between two plates separated by a distance $2 R$ actually can be obtained exactly for the case that the excluded volume per monomer $v$ equals $a^{3}$ [13]. It turns out that the confinement free energy in this case is the same as that for ideal linear polymers because of a precise cancellation between the swelling effects of excluded volume and the compression effects generated by branching. If the excluded volume interaction is now weakened, so $v<<a^{3}$, then there may be interesting cross-over behavior where we can apply our results. In scaling theory one would introduce for this case a "blob picture" where the branched polymer is subdivided into non-overlapping blobs of radius $\xi(n)$ with $n$ the number of monomers per blob. Inside each blob, excluded volume effects can be neglected so $\xi(n) \simeq a n^{1 / 4}$. The blob-size $\xi(n)$ is then determined by the condition that the excluded-volume interaction energy per blob is of the order of $k_{B} T$. This means that $v n^{2} / \xi^{3}$ should be of the order of one while the number of blobs $\mathcal{N}$ should equal $N / n$. The total confinement free energy should then be of the order of $\mathcal{N}(\xi / R)^{2}$ (in units of $k_{B} T$ ). This leads to $n \simeq\left(a^{3} / v\right)^{4 / 5}$ and $\xi / a \simeq\left(a^{3} / v\right)^{1 / 5}$. The resulting confinement free energy equals $N\left(\frac{a^{4}}{R^{2} \xi^{2}}\right)$ for $R>>\xi$ while for $R<<\xi$ it behaves as $N(a / R)^{4}$. We plan to examine this crossover using the Lifshitz equations by including the excluded volume interaction as part of the self-consistent potential.

\section{Acknowledgment}

RB would like to acknowledge support from the NSF under DMR Grant 1309423. RB and AYG thank the Aspen Center for Physics where part of this work was done with the support of the National Science Foundation under Grant No. PHY-1066293. 


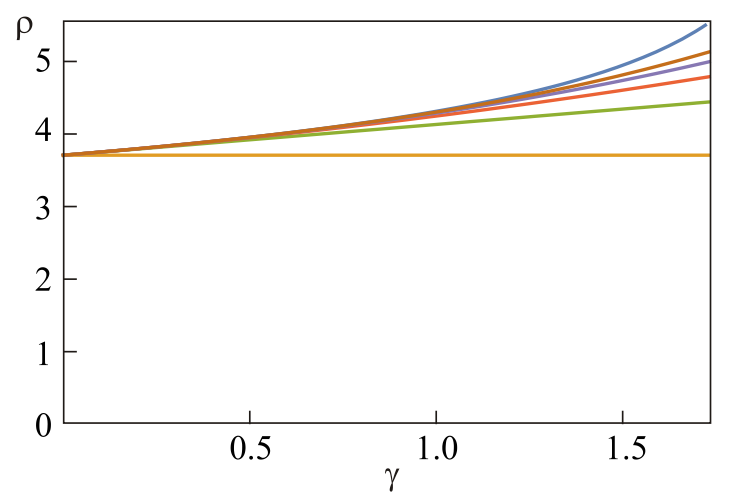

Fig. 8. (Color online) Uppermost curve is the plot of $\rho(\gamma)$ function defined in formula (15). Other curves are first five terms of the expansion in powers of $\gamma$, Eq. (A.2). The important fact is that this expansion converges rapidly and without any singularities (as long as $\gamma$ remains in the physically relevant range $\gamma<\sqrt{3}<2)$.

\section{Appendix A: Analysis of the $\rho(\gamma)$ function}

The function $\rho(\gamma)$ is defined in formula (15). It can be expanded in powers of $\gamma$ :

$$
\begin{aligned}
\rho(\gamma) & =\int_{0}^{\infty} \frac{d \eta}{\sqrt{\eta+\eta^{3}} \sqrt{1-\gamma \frac{\eta}{1+\eta^{2}}}}= \\
& =\sum_{k=0}^{\infty} \frac{(-\gamma)^{k} \Gamma(1 / 2)}{\Gamma(k+1) \Gamma(1 / 2-k)} \int_{0}^{\infty} \frac{\eta^{k-1 / 2} d \eta}{\left(1+\eta^{2}\right)^{k+1 / 2}}
\end{aligned}
$$

and all integrals in the coefficients are evaluated:

$$
\rho(\gamma)=\sum_{k=0}^{\infty} \frac{(-\gamma)^{k} 2^{k-1} \Gamma^{2}(2 k+1 / 4)}{(2 k-1) ! ! \Gamma(k+1) \Gamma(1 / 2-k)} .
$$

Furthermore, this sum can be expressed in terms of the hypergeometric function ${ }_{2} F_{1}$ :

$$
\begin{gathered}
\rho(\gamma)=\frac{\Gamma^{2}(1 / 4)}{2 \sqrt{\pi}}{ }_{2} F_{1}\left(1 / 4,1 / 4,1 / 2, \gamma^{2} / 4\right)+ \\
+\gamma \frac{\Gamma^{2}(3 / 4)}{2 \sqrt{\pi}}{ }_{2} F_{1}\left(3 / 4,3 / 4,1 / 4, \gamma^{2} / 4\right) .
\end{gathered}
$$

This formula is nice, but pretty useless, because integrals are easily computed numerically. The resulting plot of $\rho(\gamma)$, along with a few terms of expansion in powers of $\gamma$ is presented in the Fig. 8.

\section{Appendix B: Numerical methods}

The numerical method allows us also to keep the complete integral form for the operator $\hat{g}$ :

$$
\hat{g} \Psi(\mathbf{r})=\left(\frac{D}{2 \pi a^{2}}\right)^{D / 2} \int \mathrm{e}^{-\frac{D\left(\mathbf{r}-\mathbf{r}^{\prime}\right)^{2}}{2 a^{2}}} \Psi\left(\mathbf{r}^{\prime}\right) d^{D} \mathbf{r}^{\prime} .
$$

\section{Case $D=3$}

Since we consider spherically symmetric potential well, the unknown functions $\Phi(r)$ and $\Psi(r)$ are also spherically symmetric. Therefore, we first integrate over the angular degrees of freedom for the operator $\hat{g}$, which leads to

$$
\hat{g} \Psi(r)=\int_{0}^{\infty} K\left(r, r^{\prime}\right) \Psi\left(r^{\prime}\right) d r^{\prime},
$$

where

$$
K\left(r, r^{\prime}\right)=\sqrt{\frac{6}{\pi}} \sinh \left(\frac{3 r r^{\prime}}{a^{2}}\right) \frac{r^{\prime}}{a r} \mathrm{e}^{-\frac{3\left(r^{2}+r^{\prime 2}\right)}{2 a^{2}}}
$$

is a spherical kernel. Equation (6a) is then a non-linear Fredholm integral equation of the form

$$
\Phi(r)=\frac{q}{2} \int_{0}^{\infty} K\left(r, r^{\prime}\right) \mathrm{e}^{-\varphi\left(r^{\prime}\right)}\left(1+\Phi\left(r^{\prime}\right)^{2}\right) d r^{\prime} .
$$

Numerical solutions of Eq. (B.4) were obtained by a variation of Nytröms method [14] where the integral equation is discretized as a sum, starting from a set of quadrature points $\left\{r_{i}\right\}$ with a corresponding set of weights $\left\{w_{i}\right\}$ such that

$$
\Phi\left(r_{j}\right) \approx \frac{q}{2} \sum_{i} w_{i} K\left(r_{j}, r_{i}^{\prime}\right) \mathrm{e}^{-\varphi\left(r_{i}^{\prime}\right)}\left(1+\Phi\left(r_{i}^{\prime}\right)^{2}\right)
$$

which then constitutes a set of coupled non-linear equations for the unknowns $\Phi\left(r_{j}\right)$ [15-17]. Once the set $\left\{\Phi\left(r_{j}\right)\right\}$ has been obtained, one can recover a solution for $\Phi(r)$ for all values of $r$ from

$$
\Phi(r)=\frac{q}{2} \sum_{i} w_{i} K\left(r, r_{i}^{\prime}\right) \mathrm{e}^{-\varphi\left(r_{i}^{\prime}\right)}\left(1+\Phi\left(r_{i}^{\prime}\right)^{2}\right) .
$$

Surprisingly, Gaussian quadrature was not effective for discretizing our problem. We found that the CurtisClenshaw quadrature [18] was effective when combined with Newton's method to solve the coupled non-linear equations. In general integral equations can have multiple solutions, depending on the initial guess, but we found only one solution that was finite and stable. We checked the results by numerically evaluating the right hand side of equation [19], using Eq. (B.6). Differences were found to be of the order of $10^{-16}$. We also varied the quadrature point density as well as the integration upper bound, and found the smallest number of points and lowest integration upper bound that kept the peak height $\Phi(0)$ invariant [20].

The integral operator in Eq. (6b) can be discretized using Curits-Clenshaw. Equation (6b) is linear in $\Psi$ which means the quadrature equations for the unknowns $\left\{\Psi\left(r_{j}\right)\right\}$ adopt the form of a linear matrix eigenvalue equation:

$$
\hat{M}(q) \Psi=q^{-1} \Psi .
$$


The matrix $\hat{M}(q)$ is here itself a function of $q-$ and thus of the eigenvalue $1 / q$ - because of the implicit dependence of $\Phi(r)$ on $q$, which enters in Eq. (6b). This parallels the fact that for the $D=1$ analytical solution we needed to solve a Schrödinger-type equation (25) whose effective potential was dependent on the eigenvalue. To obtain solutions of Eq. (B.7) we rewrite it first in the more general form $\hat{M}\left(q_{1}\right) \Psi=q_{2}{ }^{-1} \Psi$. For a given value of $q_{1}$, one can use the numerical solution Eq. (B.6) to generate the matrix $\hat{M}\left(q_{1}\right)$. One then must find the $q_{2}$ value corresponding to the lowest eigenvalue for the linear eigenvalue equation $\hat{M}\left(q_{1}\right) \Psi=q_{2}{ }^{-1} \Psi$. The next step is to construct a plot of $q_{2}$ as a function of $q_{1}$. The value of $q^{*}$ corresponds to the intercept of the plot with the line $q_{2}=q_{1}$. Iterating $\hat{M}\left(q_{n}\right) \Psi=q_{n+1}{ }^{-1} \Psi$ causes $q_{n+1}$ to diverge away from the solution, which unfortunately means that the correct $q_{1}$ must be guessed a'priori. Fortunately, the correct $q_{1}$ followed a pattern that could be determined empirically. The largest $q_{1}$ possible that solves $\Phi$ also solves $\Psi$. Solving $\Phi$ with too large a $q_{1}$ leads to failure of convergence of Newton's method. We define $q_{\max }$ to be the largest possible $q$, as well as the numerically correct answer. To find $q_{\text {max }}$, we employ a binary search pattern in the range $\left(q_{\text {low }}\right.$, $\left.q_{\text {high }}\right) . q_{\text {low }}$ is selected so $q_{\text {low }}<q_{\text {max }}$, and $\Phi$ will converge. $q_{\text {high }}$ is selected such that $q_{\text {high }}>q_{\max }$, and $\Phi$ will diverge. We divide this range in half, and find a new smaller range that has boundary conditions (converging $q_{\text {low }}$ and diverging $q_{\text {high }}$ ). This procedure is iterated recursively until any desired precision.

\section{Case $D=1$}

Solving the $D=1$ case numerically is more difficult than the $D=3$ case, because quadrature points must be chosen for both positive and negative points on the $x$ axis, whereas in 3D spherical quadrature points need only lay on $r>0$. This introduces twice as many coupled equations in $1 \mathrm{D}$, and takes much longer to solve. To get around this, It is useful to symmetrize the $1 \mathrm{D}$ kernel, by using the fact that $\Phi(-x)=\Phi(x), \varphi(-x)=\varphi(x)$ and $\Psi(-x)=\Psi(x)$. In other words, all the functions for which we are solving are even about the origin, so we only need to solve the problem for $x>0$. To this end, we can split the $1 \mathrm{D} \hat{g}$ operator at the origin, adjust bounds, and write a new kernel defined for $x>0$ as:

$$
K\left(x, x^{\prime}\right)=\frac{2}{\sqrt{2 \pi a^{2}}} \mathrm{e}^{-\frac{x^{2}+x^{\prime 2}}{2 a^{2}}} \cosh \left(\frac{x x^{\prime}}{a^{2}}\right),
$$

which is the $1 \mathrm{D}$ analog of the spherical kernel (B.3).
1. I.M. Lifshitz, Zh. Eksp. Teor. Fiz. 55, 2408 (1968) [I.M. Lifshitz, JETP 28, 1280 (1969)].

2. I.M. Lifshitz, Selected Scientific Papers: Electron Theory of Metals. Polymers and Biopolymers, Nauka Publishers, Moscow (1994).

3. A.Y. Grosberg and A.R. Khokhlov, Adv. Polymer Science 196, 189 (2006).

4. P.-G. de Gennes, Biopolymers 6, 715 (1968).

5. L. Fang, W. Gelbart, and A. Ben-Shaul, J. Chem. Phys. 135, 155105 (2011).

6. A. Gopal, Z. Zhou, C.M. Knobler, and W.M. Gelbart, RNA 18, 284 (2012).

7. R. Garmann, M. Comas-Garcia, A. Gopal, C.M. Knobler, and W.M. Gelbart, J. Mol. Biol. 426, 1050 (2013).

8. R.F. Bruinsma, M. Comas-Garcia, R.F. Garmann, and A.Y. Grosberg, Phys. Rev. E 93, 032405 (2016).

9. A.Y. Grosberg, A. Gutin, and E. Shakhnovich, Macro-molecules 28, 3718 (1995).

10. B.H. Zimm and W.H. Stockmayer, J. Chem. Phys. 17, 1301 (1949).

11. These relations are for $D=1$. For general $D$, one must replace $\frac{1}{(k+1)(2 k+1)} \rightarrow \frac{D}{(k+1)(2 k+D)}$ in the first term of formula (19b).

12. Interestingly, according to its definition, $A(\gamma)$ has a maximum at $\gamma \approx 0.838$ where $A \approx 87.62$. This value for $\gamma$ is surprisingly close to the $\gamma=\sqrt{3} / 2 \approx 0.866$ obtained in the harmonic approximation with $A \approx 87.59$. The value $A \simeq 94.2$ obtained by the fit is thus probably not physical.

13. R. Ghafouri, J. Rudnick, and R. Bruinsma, Europhys. Lett. 82, 46003 (2008).

14. K.E. Atkinson, The Numerical Solution of Integral Equations of the Second Kind, Cambridge University Press (1997), Vol. 4.

15. S. Yousefi and M. Razzaghi, Math. Comput. Simulat. 70, 1 (2005).

16. S. Rahbar and E. Hashemizadeh, in: Proceedings of the World Congress on Engineering (2008), Vol. 2, p. 933.

17. Y. Mahmoudi, Appl. Math. Comput. 167, 1119 (2005).

18. P.J. Davis and P. Rabinowitz, Methods of Numerical Integration, Courier Corporation (2007).

19. We used Mathematica's FindRoot implementation.

20. We use an upper bound or $R / a=10$, and a quadrature point density of 0.2 , or $1 / 0.2$ points for every unit distance $a$. 